International Journal of Advanced Trends in Computer Science and Engineering

Available Online at http://www.warse.org/IJATCSE/static/pdf/file/ijatcse971032021.pdf

https://doi.org/10.30534/ijatcse/2021/981032021

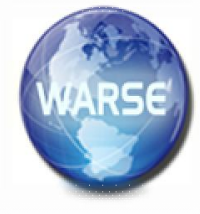

\title{
Home Security
}

\author{
Shanty Thomas ${ }^{1}$, Ansil $\mathbf{H}^{2}$, Seethu George ${ }^{3}$ \\ ${ }^{1}$ Department of Computer Science and Engineering, AISAT,Kalamassery, shantythomasav@gmail.com \\ ${ }^{2}$ Department of Computer Science and Engineering, AISAT,Kalamassery, ansilhaneef77@ gmail.com \\ ${ }^{3}$ Department of Computer Science and Engineering, AISAT, Kalamassery, seethugeorge @ aisat.ac.in
}

\begin{abstract}
The security and privacy of our homes and residences are very important these days. This can protect our homes and valuables, thereby keeping our family safe from potential intrusion by thieves. The purpose of this project is to allow entry of people and vehicles to a home only on owner's approval. By designing and installing a smart home security system we can achieve this. The security system will be equipped with camera modules and sensors which can detect the faces of people and vehicles entering our home. Haar Cascade algorithm which is an object detection algorithm identifies real time faces in a video and Optical Character Recognition recognizes vehicles based on the number plate. An android application will be used to control things happening around the home, using which the users of the application can view requests from people or vehicles entering our home. Only the recognized or entry accepted people and vehicles will be allowed to enter our home. The process will be repeated in all cases. The system has a good accuracy level too. Thus, security is guaranteed.
\end{abstract}

Key words: Face recognition, Haar cascade, Home security, Optical Character Recognition, Security, Vehicle detection.

\section{INTRODUCTION}

Almost every home in our neighbourhood is a target of intrusion whether we live in the city or country. Thus, a security system plays a very crucial role in every home. Previously, home security systems had alarms that would go off when somebody intrudes into our home but today's security systems can do much more than that. Therefore, the main objective of our project is to design a system that can alert the owner and other people in a home of an intruder by sending alerts to them through an android application. The owner also has the authority to deny requests coming in. Thus, the gates of the home will remain closed until entry is allowed. This system will help users to protect their homes by placing this system on the gates of the home and the doors of the home, thereby monitoring real time activities through their smartphones. Many home security systems only check the people entering a home. But our security system also identifies the vehicles coming in. O u r system uses face recognition and vehicle recognition to verify the people and vehicles entering the home. Only the verified people and vehicles will be allowed entry.
Others are denied of entry and the gates remain closed. Thereby, ensuring security.

The existing face recognition systems use Local Binary Pattern, Viola Jones algorithm, etc. to recognize faces. But our system uses Haar Cascade algorithm for face recognition which has a comparatively higher accuracy than other algorithms and for vehicle identification, the vehicles are distinguished based on their registration number in their license plates. In this paper, we focused on ensuring real time security keeping the owner informed about the security status of their home. The developed system alerts the owner of the current status of the home and he can take necessary actions accordingly.

This paper is organized as follows: The first section discusses about the introduction of security system using face recognition and vehicle identification. The second section briefs the focus of the paper. The third section talks about the methodologies used in the paper. The next section explains the experimental results and observations. And finally, sections five and six draws the conclusion and future enhancement.

\section{LITERATURE REVIEW}

Various methodologies have been used in different security systems. Face Detection and Recognition Using Open CV [1] proposes using a device that could classify photos using RAND tablet whose input is horizontal and vertical coordinates on a grid that emits electromagnetic pulses. The training modules train the system using Haar Cascade algorithm. Facial Detection and Recognition using Open CV Library [2] suggests a built-in face detection, face tracking and face recognition Open CV library that has about $90-95 \%$ accuracy level. Human face detection algorithm via Haar cascade classifier combined with three additional classifiers [3] makes use of three classifiers for skin hue histogram matching, eye detection and finally mouth detection. Under different illuminations and angles, the test results on different images of people in both training set and test set proved that this method is an effective one. A Detailed Analysis of Optical Character Recognition Technology [4] proposes extracting text from printed or hand written documents. A Survey on Optical Character Recognition System [5] uses OCRA and OCRB for improved accuracy rates. They also suggest document image analysis, multi-lingual, handwritten and OmniOCR fonts. A Comprehensive Analysis of Image Edge 
Detection Techniques [6] proposes extracting the edges in an image and the comparative analysis is performed among these techniques. Number plate recognition and document verification using feature extraction OCR algorithm [7] applies feature extraction optical character recognition algorithm for character segmentation. Automatic Number Plate Recognition [8] uses image processing concept to analyses the printed characters on the segmented license plate. Automatic Vehicle License Plate Recognition System Based on Image Processing and Template Matching Approach [9] proposes using image processing as well as template matching approach where captured images of vehicles will be the input which will be further processed to segment the number plate.

\section{METHODOLOGY}

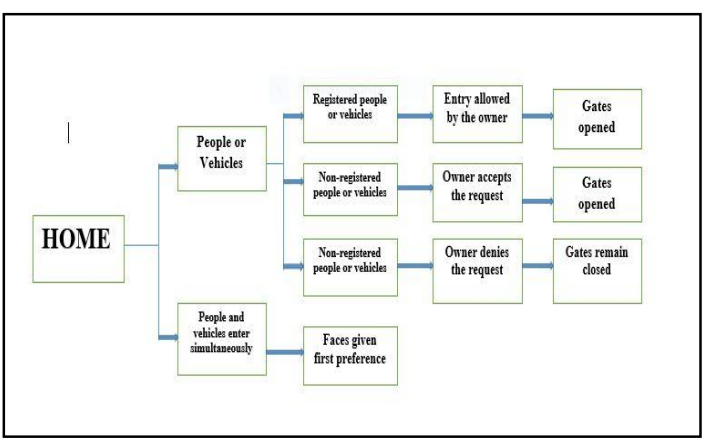

Figure 3.1: Flowchart of the system

The system is designed in such a way that when registered people or vehicles enter our home, they are automatically allowed entry into the home. But, when non-registered people or vehicles arrive, their entry depends upon the decision of the owner. When both people and vehicles arrive at the same time, people are given preference as demonstrasted in Figure 3.1.

\subsection{Face Recognition for the Identification of People}

As shown in Figure 3.3, Haar Cascade algorithm comprises of the following major steps:
a. Calculating Haar features.
b. Creating Integral Images.
c. Using AdaBoost.
d. Implementing cascading classifiers.

\subsubsection{Haar Features}

The first step is to collect the Haar features which are calculations that are performed on adjacent rectangular regions at a specific location in a detection window. Summing the pixel intensities in each region and calcula- ting the differences between the sums is the calculation part. But the features become difficult to determine when it comes to action.

\subsubsection{Creating Integral Images}

The calculations of the above said Haar features are made fast by using integral images. In this stage, it creates sub-rectangles and creates array references for each of those sub-rectangles instead of computing at every pixel which is then used to compute the Haar features. Since, the only features that are important are those of the object, most of the Haar features will be immaterial. To determine the best features that represent an object from thousands of Haar features is done by AdaBoost training.

\subsubsection{AdaBoost Training}

AdaBoost actually chooses the best features and trains the classifiers using them. A large number of Haar features are actually needed for accuracy to form a strong classifier since there are weak classifiers. The final step of cascading classifiers combines the weak learners into strong learners.

\subsubsection{Implementing Cascading Classifiers}

Susceptible learners are trained using boosting which allows for a distinctly correct classifier from the mean prediction of all vulnerable learners. Based on this prediction, the classifier classifies them as positive or negative by checking whether there is an object found or move on to the next region. Stages are designed to reject negative samples as rapid as possible because a majority of the windows do not incorporate anything of interest. It's vital to maximize a low false negative rate since classifying an object as non-object will significantly impair our object detection algorithm. Haar Cascade is certainly one of the many algorithms which can be used for object detection. An important point to be noted about this algorithm is that it reduces the false negative rate, so the hyper-parameters should be treated as such when training our model.

\subsection{Vehicle Recognition for the Identification of unknown Vehicles Entering Our Home}

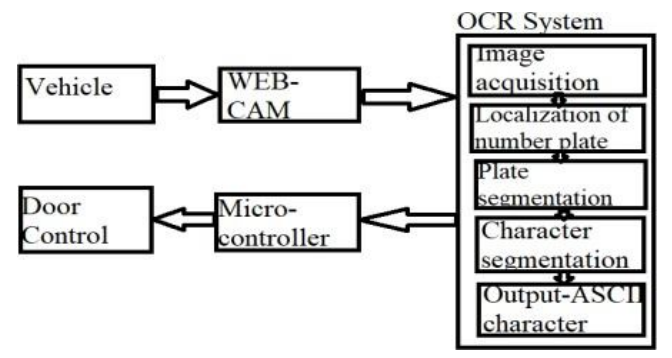

Figure 3.2: OCR Algorithm 


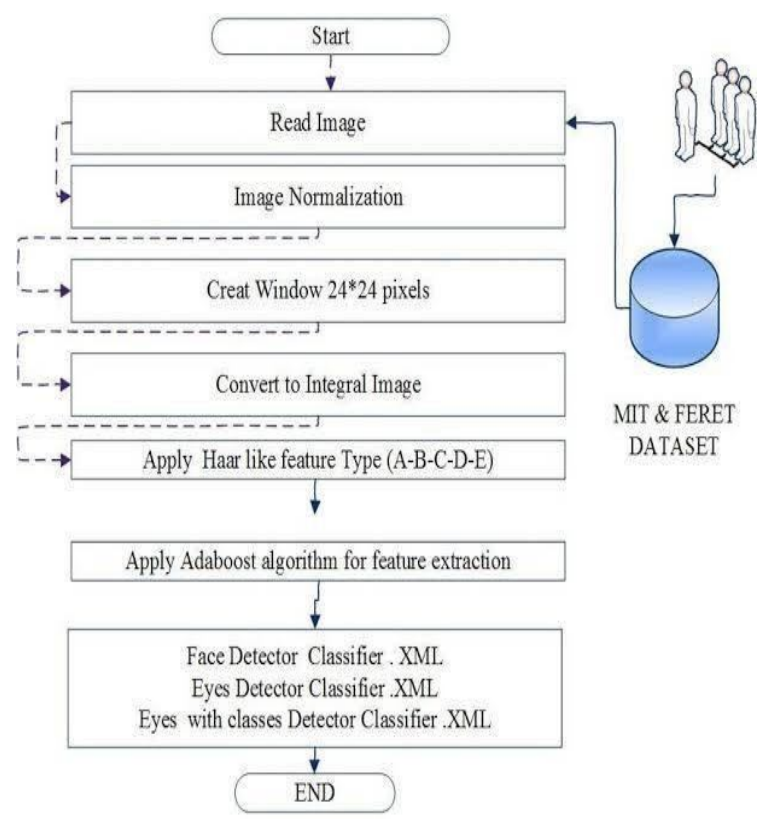

Figure 3.3: Haar Cascade Algorithm

The major steps involved in OCR algorithm for number plate recognition as shown in Figure 3.2 are as follows:

a. Extraction of character boundaries from image.

b. Building a convolutional neural network for remembering the character images.

c. Load trained Convolutional Neural Network OCR model.

d. Consolidating Convolutional Neural Network predictions of characters.

\subsubsection{Optical Scanning from Image}

First of all, we select a document containing text information. For extracting character boundaries, we create a boundary to each character in the image using open CV contours method. The extracted textual content characters should be labelled with the unique individual name associated with it. In the pre-processing step, the raw data relying at the facts acquisition type is subjected to a number of preliminary processing steps to make it usable within the descriptive ranges of character analysis. The resulting image may contain noise. The width of line is reduced by filling which eliminates small breaks, gaps and holes in the digitized characters.

\subsubsection{Building a Character Recognition Model}

The first model trains the individual character images with direct classification for predicting the images with soft max classification of character categories whereas the second model is the same with the second last layer acting as a predictor which calculates the embedding of specified flatten neurons.

\subsubsection{Load Trained Convolutional Neural Network OCR Model}

This step involves pre-processing of image into unique phrase associated contours and letter contours accompanied through prediction and consolidating in keeping with letter and word associated contours in a photograph. After training the model, the pre-trained optical character recognition model can be saved and loaded.

\subsubsection{Testing and Consolidating Predictions of Optical Character Recognition}

The consolidation of predictions involves assigning specific ID to each word in image thereby consolidating all predictions in a sorted series of specific word related contour and letters associated word.

\section{RESULTS}

This system is used to verify people and vehicles entering a home for security purpose. Various camera modules will be placed at the gates of the home and at the Front door of the home. In the first scenario, using face recognition, when the system detects a face in the real time video footage, the face will be checked for verification whether it has already been registered in the system or not. If it is a registered one, a character from the checked for verification whether it has already been registered in the system or not. If it is a registered one, a character from the android device in the automatic gate via Bluetooth. When this character is received, the motor in the gates will be rotated and the gates will be automatically opened. In the second scenario, whenever a vehicle enters our home and if it has been already registered in the system, then the system detects the number plate in the vehicle, verifies it and a character sent from the android device sent to the gates will make it open. Next, if a non-registered person or vehicle enters, then the android device will get a notification regarding the same and the user of the application can grant access to the above said person or vehicle by sending a character via Bluetooth to the gate. Or else he can deny the access so that the gates remain closed also. The various people and vehicle entry requests can be checked by the user at any time as shown in Figure 4.1. Thus, unknown persons or vehicles are refrained from entering our home.

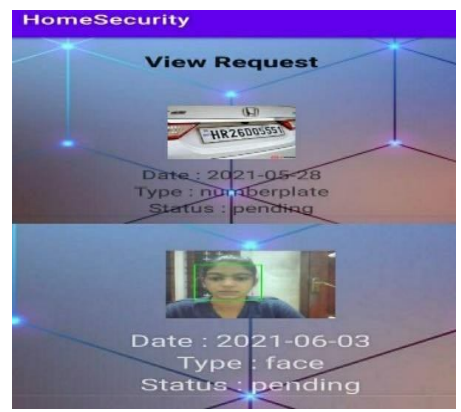

Figure 4.1: Request portal of home security 


\section{CONCLUSION}

Security is an imperative part of any enterprise. This work is most particularly for the security of homes and residences. In this paper, a system that identifies realtime face and vehicle recognition using Haar Cascade algorithm and Optical Character Recognition technology was proposed for the security of homes. The proposed system uses Haar Cascade algorithm for the recognition of faces in the real-time video which is good at detecting edges and lines. This makes it effective in face detection making it able to recognize objects with clear edges and lines. Optical Character Recognition technology is used to read number plates of the vehicles which extracts the characters of the number plate which will be further processed for classification. Thus, only the system registered people and vehicles enter our home.

\section{LIMITATION AND FUTURE STUDIES}

The main drawback of the system is that different face angles can throw off face recognition's and vehicle recognition's reliability. Occlusion being another problem in the current scenario. The future scope of this system is developing systems using OCR for daily practical use incorporating mobile phones. Automatic book reader, receipt reader is some of the future scopes of OCR.

\section{REFERENCES}

1. Paliy,Face detection using Haar-like features cascade and convolutional neural network In 2008 International Conference on "Modern Problems of Radio Engineering, Telecommunications and Computer Science" (TCSET),IEEE 2008.

2. Priadana and M.Habibi, Face Detection using Haar Cascades to Filter Selfie Face Image on Instagram In 2019 International Conference on Artificilal Intelligence and Information Technology(ICAIIT),IEEE 2019.

3. R.Yustiawati et al, Analyzing of different features using Haar Cascade Classifier In 2018 International Conference on Electrical Engineering and Computer Science (ICECOS),IEEE 2018.

4. L.Cuimei,Q.Zhiliang J.Nan and W.Jianhua, Human face detection algorithm via Haar. cascade classifier combined with three additional classifiers In $2017 \quad 13^{\text {th }}$ IEEE.International Conference on Electronic Measurement \& Instruments (ICEMI),IEEE 2017.

5. B.Tejas, D.Omkar , D.Rutuja,K.Prajakta and P.Bhakti, Number plate recognition and document verification using feature extraction OCR algorithm In 2017 Internatio al Conference on Intelligent Computing and Control Systems(ICICCS),IEEE 2017.

6. A Kashvan ,B.Suresh ,A.Patil ,S.Sharma and A.Jaiswal, Automatic Number Plate Recognition In 2018 International Conference on Advances in Computing,Communication Control and Networking ICACCN),IEEE 2018.
7. S.S.Omran and J.A.Jarallah, Iraqi car license plate recognition using OCR In 2017 Annual Conference on New Trends in Information \& Communications Technology Applications (NTICT), IEEE 2017.

8. V. Kakani, D. Gandhi and S. Jani, Improved OCR based automatic vehicle number plate recognition using features trained neural network In 2017 8th International Conference on Computing, Communication and Networking Technologies (ICCCNT), IEEE 2017.

9. Agbemenu , A.S ,Yankey,J and Addo ,E.O, 2018 An automatic number plate recognition system using opencv and tesseract ocr engine, International Journal of Computer Applications, 180,pp.1-5.

10. CH.Y.Lu CH.SH.Zhang \& F.Wen(1999), Regional Feature based Fast Human Face Detection ,J. Tsinghua Univ.

11. M.K.Rusia , D.K Singh and M.A Ansari ,Human Face Identification using LBP and Haar- like Features for Real Time Attendance Monitoring In 2019 Fifth International Conference on Image Information Processing (ICIIP), IEEE 2019.

12. T.Mantoro, M.A Ayu and Suhendi, Multi Faces Recognition Process Using Haar Cascades and Eigenface Methods, In $6^{\text {th }}$ International Conference on Multimedia Computing and Systems(ICMCS),IEEE 2018. 